\title{
Die Kopplung elektrotechnischer und strukturdynamischer Domänen zu einem NVH-Systemmodell eines elektrischen Antriebsstrangs
}

\author{
M. Jaeger(D, P. Drichel, M. Schröder, J. Berroth, G. Jacobs, K. Hameyer
}

\begin{abstract}
In dieser Arbeit wird am Beispiel eines Antriebsstrangs eines Serien-Elektrofahrzeugs eine Methode vorgestellt, welche das elektrotechnische mit dem strukturdynamischen Teilsystem rückwirkungsbehaftet recheneffizient verbindet. Das transiente, nichtlineare Systemmodell berücksichtigt auf elektrotechnischer Seite Regelung, Leistungselektronik und das Verhalten der elektrischen Maschine unter dem Einfluss von Fertigungsabweichungen und strukturdynamischen Rückwirkungen wie dynamischer Exzentrizität, Torsion und Biegung des Rotors. Auf strukturdynamischer Seite ist der vollständige Antriebsstrang inklusive. Wellensystem, Getriebe und Gehäuse in einer elastischen Mehrkörpersimulation zur Berechnung transienter Vorgänge im Zeitbereich abgebildet. Die Methode erlaubt die Berechnung des nichtlinearen, akustischen Systemverhaltens und wird anhand von Prüfstandsmessungen validiert. Die Möglichkeiten, durch Variantenrechnungen die Sensitivitäten des NVH-Verhaltens zu untersuchen oder Komponenten im Auslegungsprozess auf Systemebene zu optimieren, werden aufgezeigt.
\end{abstract}

Schlüsselwörter: Simulation; Akustik; elektrische Maschine; Antriebsstrang; Kraftanregung; Mehrkörpersimulation

\section{The coupling of electrotechnical and structural dynamics domains for a NVH system model of an electrical drive train.}

In this paper, an approach is presented using the example of a drive train of a series-produced electric vehicle which connects the electrotechnical domain with the structural dynamics domain in an efficient way including retroactive effects. On the electrotechnical side, the transient, nonlinear system model considers control, power electronics and the behavior of the electrical machine under the influence of manufacturing deviations and structural dynamic feedback such as dynamic eccentricity, torsion and bending of the rotor. On the structural dynamics side, the complete drive train including shaft system, gearbox and housing is represented in an elastic multibody simulation in the time domain to simulate transient processes. The approach allows the calculation of the nonlinear acoustic system behavior and is validated by measurements. The possibilities to study the sensitivities of the NVH behavior by variant calculations or to optimize components in the design process on system level are shown and discussed.

Keywords: simulation; acoustics; electrical machine; drive train; force excitation; multibody simulation

Eingegangen am 21. Februar 2020, angenommen am 29. April 2020, online publiziert am 19. Mai 2020

(c) The Author(s) 2020

\section{Einleitung}

Elektrische Maschinen sind seit ihrer Erfindung bekannt dafür, tonale, störende Geräusche zu erzeugen [1], [2]. Die Beschreibung der elektromagnetischen Anregungskräfte durch JORDAN [3] als räumlich und zeitlich wandernde Kraftwellen war wegweisend für weitere Arbeiten zur Verbesserung des akustischen Verhaltens und Auslegungsliteratur el. Maschinen [4]. Die aufkommende Leistungselektronik führte zu neuen Möglichkeiten und Herausforderungen (vgl. z. B. [5], [6]). Einen Überblick über das im 20. Jahrhundert erarbeitete Wissen der Akustik el. Maschinen bietet z. B. die Arbeit von GIERAS [7].

In den letzten Jahren sind die Ansprüche an das akustische Verhalten el. Antriebe gestiegen, u.A. durch den Automobilsektor. Aktuelle Anforderungen bzgl. Lärm, Vibrationen und 'Unangenehmheit' (NVH, engl. Noise, Vibration, Harshness) beinhalten nicht nur die Reduktion der Geräuschemissionen, sondern einen zielgerichteten Entwurf hinsichtlich einer z. B. als komfortabel, kräftig oder markenspezifisch empfundenen Klangcharakteristik, um dem subjektiven Kaufverhalten gerecht zu werden [8], [9]. Hierfür sind effiziente
Berechnungsmethoden erforderlich, welche in frühen Entwicklungsphasen das Geräuschverhalten des el. Antriebssystems in zweckmäBiger Näherung vorhersagen können.

Aktuelle akustische Simulationsketten ([10], [11], [12]) verbinden den in den bisherigen Arbeiten zentralen Aspekt der Schwingungsanregung, welcher die Bereiche Schaltungssimulation, Feldund Kraftberechnung umfasst, mit dem strukturdynamischen Ver-

Jaeger, Markus, Institut für Elektrische Maschinen (IEM), RWTH Aachen University, Schinkelstraße 4, 52062 Aachen, Deutschland

(E-Mail: markus.jaeger@iem.rwth-aachen.de); Drichel, Pascal, Institut f. Maschinenelemente u. Systementwicklung (MSE), RWTH Aachen University, Schinkelstraße 10, 52062 Aachen, Deutschland (E-Mail: pascal.drichel@mse.rwth-aachen.de); Schröder, Michael, Institut für Elektrische Maschinen (IEM), RWTH Aachen University, Schinkelstraße 4, 52062 Aachen, Deutschland; Berroth, Joerg, Institut f. Maschinenelemente u. Systementwicklung (MSE), RWTH Aachen University, Schinkelstraße 10, 52062 Aachen, Deutschland; Jacobs, Georg, Institut f. Maschinenelemente u. Systementwicklung (MSE), RWTH Aachen University, Schinkelstraße 10, 52062 Aachen, Deutschland; Hameyer, Kay, Institut für Elektrische Maschinen (IEM), RWTH Aachen University, Schinkelstraße 4, 52062 Aachen, Deutschland 


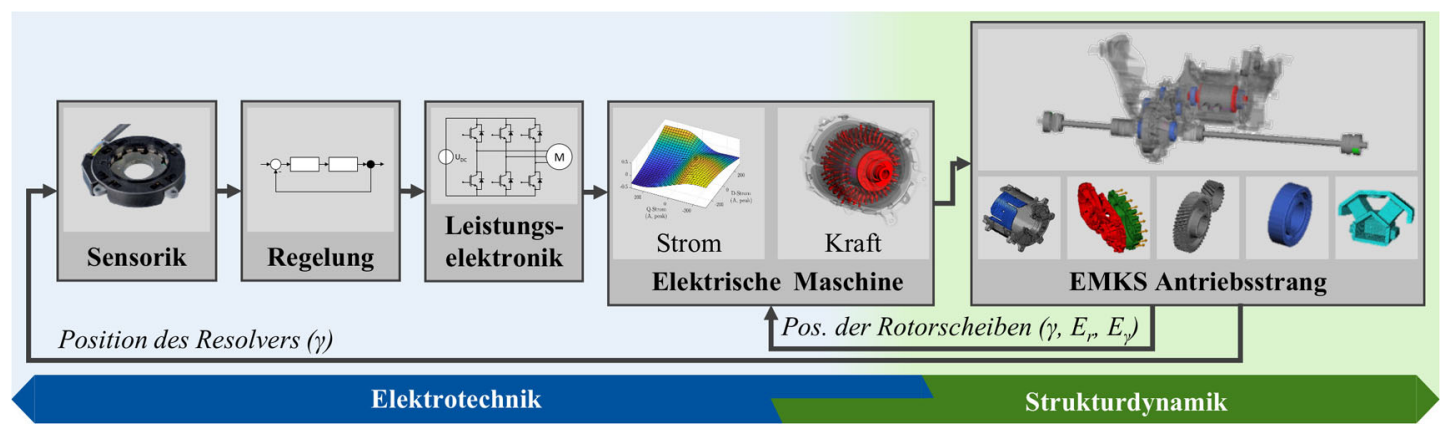

Abb. 1. NVH-Systemmodell

halten, welches z. B. mit Methoden der Transferpfadanalyse (vgl. [13]) beschrieben werden kann. In [12] werden Anregungskräfte an den Statorzähnen sowie an den Lagerstellen berechnet, um sie in einem anschließenden Schritt in einer Forced-Response Analyse auf die Statorstruktur aufzuprägen. Das Vorgehen steht beispielhaft für die weiteren referenzierten Ansätze, welche durch die vorwärts gerichtete Untersuchung die Rückkopplung des mechanischen Systems auf das elektromagnetische Verhalten vernachlässigen.

Zur Kopplung ist die Berechnung des strukturdynamischen Verhaltens im Zeitbereich erforderlich, z. B. mittels elastischer Mehrkörpersimulation (EMKS). Dies ermöglicht weiterhin die Abbildung von Nichtlinearitäten. In der EMKS werden die Gehäuse und Wellen typischerweise als elastische Körper durch Methoden der modalen Reduktion (vgl. [14], [15], [16]) ordnungsreduziert abgebildet. Im Gegensatz zu einem direkten Ansatz mittels Finite-Elemente Methode (FEM) ist erst so die Berechnung großer Systeme im relevanten Frequenzbereich in sinnvollen Zeitspannen möglich. In [17] wird eine EMKS mit Fokus auf das strukturdynamische Verhalten der von Kompositmaterialien geprägten el. Maschinen vorgestellt, im Vorgängerprojekt dieser Arbeit [18] und in [19] die EMKS eines Fahrzeugantriebsstrangs, jedoch jeweils mit Kraftanregungsspektren aus der FE unter Vernachlässigung von Rückwirkungen.

Die vorliegende Arbeit stellt die Verknüpfung der dargestellten Ansätze aus den Domänen Elektrotechnik und Strukturdynamik zu einem rückwirkungsbehafteten, physikalisch gekoppelten Systemmodell dar (Abb. 1). Dieser Ansatz ist in der Lage, die Wechselwirkung von Regelung und Leistungselektronik mit der Struktur inkl. maßgeblicher Nichtlinearitäten und Fertigungsabweichungen physikalisch motiviert abzubilden und erlaubt Vorhersagen des $\mathrm{NVH}$ Verhaltens, so dass z. B. eine gezielte Einzelteiloptimierung im Gesamtsystem möglich ist. Der Rechenaufwand, welcher in den Vorarbeiten als Grund für die Vernachlässigung der Kopplung genannt wird (vgl. z. B. [10]), wird begrenzt durch eine Feld-SchaltungsSimulation mit parallelisierter Vorabberechnung (Kap. 2.2, vgl. [20], [21]), die numerische Entkopplung der Domänen (Kap. 2.3) sowie vorverarbeiteten und nach [11] ordnungsreduzierten Anregungskräften mit Ausnutzung der verbleibenden Symmetrien (Kap. 2.4). Der Fokus der Arbeit liegt auf der elektrotechnischen Domäne und wird abgerundet durch eine kurze Darstellung der EMKS des Antriebsstrangs (Kap. 2.5). Das Systemmodell wird an Prüfstandsmessungen des Antriebsstrangs verifiziert und die Vorhersagegüte bestimmt. Die Arbeit schließt mit Anwendungsbeispielen um exemplarisch das Potential der Methode zur Vorhersage des NVH-Verhaltens aufzuzeigen.

\section{Modellbildung}

\subsection{Einflüsse auf die Kraftanregung}

Im Körperschall der einzelnen el. Maschine (Abb. 2) sind neben den Hauptordnungen (Vielfache von Drehfrequenz und Polpaarzahl/Nutzahl, vgl. [3]) und dem sogenannten „PWM-Fächer" Nebenbänder geringerer Amplitude erkennbar, welche nach GIERAS [7] auf Drehschwingungen und Exzentrizitäten, d.h. Fertigungsabweichungen zurückzuführen sind.

Nach dem MAXWELLSCHE Spannungstensor sind die magnetischen Kräfte direkt proportional zum Quadrat der magnetischen Flussdichte $B$. Die bestimmenden Parameter des magnetischen Flusses, deren dominante Nichtidealitäten betrachtet werden, sind erstens die Erregung, welche aus dem Stromfluss in der Wicklung resultiert, zweitens die Erregung durch die Permanentmagneten, sowie drittens der magnetischen Leitwert des Luftspaltes.

- Die Stromberechnung wird in Kap. 2.2 beschrieben.

- Die Streuung der Magnetisierung der Permanentmagnete liegt laut Herstellerdatenblatt und eigener Messung in der Größenordnung $\pm 5 \%$ und wird in der Feldberechnung berücksichtigt.

- Den dominanten Einfluss auf den Luftspalt stellt die Exzentrizität durch Verlagerung oder Biegung der Welle dar. In verschiedenen analytischen [22], [23], [24] und numerischen [25] Arbeiten wurde u.a. dynamische Exzentrizität als Ursache von Nebenbändern identifiziert und wird daher auch hier modelliert.

\subsection{Feld- und Stromberechnung}

Zur Modellierung des elektrischen Systems werden die Teilbereiche der Feldberechnung und der Schaltungssimulation nach dem Ansatz aus [20] verbunden und aufgrund des Unterschieds der Zeitkonstanten entkoppelt berechnet. Im Fall einer PMSM mit vergrabenen Permanentmagneten stellt die Betrachtung des magnetischen Feldes als stationär und die damit verbundene Vernachlässigung von zeitabhängigen Effekten wie Wirbelströmen und Stromverdrängung eine zulässige Näherung dar. Die somit lediglich von den Zustandsvariablen Strom und Rotorposition abhängige Feldberechnung kann parallelisiert vorab durchgeführt und die Ergebnisse als konzentrierten Größen in Kennfeldern abgelegt werden.

Die Feldberechnung der PMSM mit Geometrieabweichungen führt in 3D zu einer großen Anzahl an Freiheitsgraden. Eine Reduktion wird mittels der Multi-Slice Methode erreicht (vgl. [26], [27]), welche das Verhalten durch Diskretisierung des Rotors in gegeneinander verschiebbare Scheiben mit in axialer Richtung konstanten Eigenschaften annähert. Wird das gleiche Kennfeld für alle Scheiben verwendet, können Unterschiede der Magnetisierungsabweichungen in den Anregungskräften durch einen Drehwinkeloffset um vielfache einer Polteilung angenähert werden. Die Abbildung 


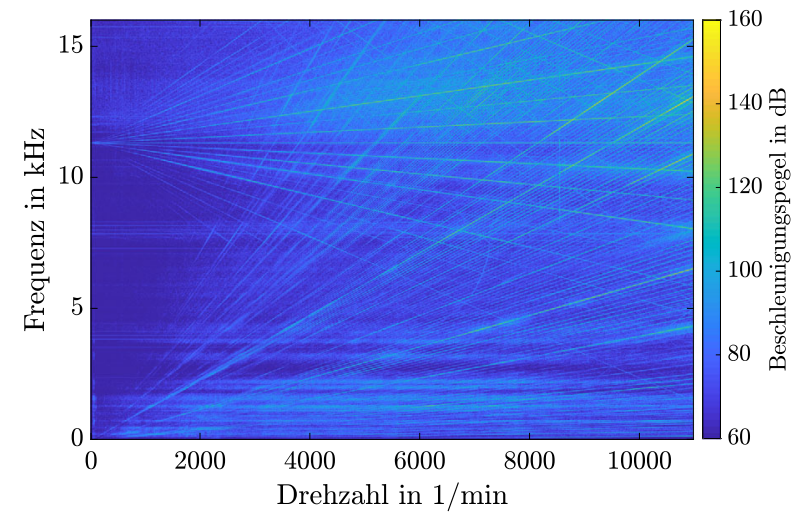

Abb. 2. Gemessener Körperschall radial auf der elektrischen Maschine bei einer PWM-Frequenz von 11,3 kHz

der Exzentrizität der Scheiben erweitert in 2D den Zustandsraum um ihren Radius $r_{E x}$ und Winkel $\phi$.

Durch die Symmetrie der PMSM reduziert sich die Anzahl der erforderlichen Feldberechnungen. Um die Kräfte an allen Zähnen zu erhalten wird ein Vollmodell verwendet. Die Statorgeometrie weist Symmetrie durch die Nutteilung auf. Bei Exzentrizität ergeben sich aus erstens der Statorposition um max. eine Nutteilung (Exz. Richtung Nut oder Zahn), zweitens eine volle Rotordrehung sowie drittens den Radius $r_{E x}$ die drei Dimensionen, welche alle Fälle abdecken. Das Feld jeder geometrischen Position wird für alle zu erwartenden Stromkombinationen berechnet. Bei Vernachlässigung von Magnetisierungsabweichungen wiederholt sich die Rotorgeometrie nach einer Polteilung, so dass der max. zu berechnende Rotordrehwinkel $\gamma$ sich auf diesen Wert reduziert.

Die Schaltungssimulation basiert auf dem Verfahren aus [11]. Das Prinzip und die Erweiterung um die Multi-Slice Methode werden hier kurz dargestellt (vgl. [28]). Die vom dreisträngigen Wechselrichter gestellten Spannungen werden zur Berechnung der Stromreaktion der PMSM verwendet und die Ströme der Schaltungssimulation im nächsten Zeitschritt eingeprägt. Das Modell basiert auf der allgemeinen Spannungsgleichung der Synchronmaschine (Gleichung 1) im rotorfesten dq-Koordinatensystem mit dem ohmschen Widerstand $R$, sowie den von Rotorposition $\gamma$ und Strom $i_{\mathrm{d}}, i_{\mathrm{q}}$ abhängigen GröBen: Der von den Permanentmagneten verursachte Fluss $\psi_{\mathrm{f}}$, den Selbstinduktivitäten $L_{\mathrm{dd}}$, $L_{\mathrm{qq}}$ und der Koppelinduktivität $L_{\mathrm{dq}}$.

$$
\begin{aligned}
& u_{d}=R_{\mathrm{dd}} i_{\mathrm{d}}+\frac{\mathrm{d}}{\mathrm{d} t}\left[\psi_{\mathrm{fd}}+L_{\mathrm{dd}} \cdot i_{\mathrm{d}}+L_{\mathrm{dq}} \cdot i_{\mathrm{q}}\right] \\
& u_{\mathrm{q}}=R_{\mathrm{qq}} i_{\mathrm{q}}+\frac{\mathrm{d}}{\mathrm{d} t}\left[\psi_{\mathrm{fd}}+L_{\mathrm{dq}} \cdot i_{\mathrm{d}}+L_{\mathrm{qq}} \cdot i_{\mathrm{q}}\right]
\end{aligned}
$$

Die Ausführung der zeitlichen Ableitung führt zu umfangreichen Gleichungen. Es ist daher zweckmäßig, die Kennfeldparameter aus der Feldberechnung und den aus dem vorherigen Zeitschritt bekannten Strom in den Abkürzungen $A, B, C, D, X$ und $Y$ zusammen zu fassen, welche hier aus Platzgründen nicht dargestellt sind.

$$
\begin{aligned}
& u_{d}=\frac{\mathrm{d} i_{\mathrm{d}}}{\mathrm{d} t} \cdot A+\frac{\mathrm{d} i_{\mathrm{q}}}{\mathrm{d} t} \cdot B+X \\
& u_{q}=\frac{\mathrm{d} i_{\mathrm{d}}}{\mathrm{d} t} \cdot C+\frac{\mathrm{d} i_{\mathrm{q}}}{\mathrm{d} t} \cdot D+Y
\end{aligned}
$$

Weiter abkürzend wird im folgenden die Tensorschreibweise verwendet.

$$
u=\left[\begin{array}{cc}
A & B \\
C & D
\end{array}\right] \cdot \frac{\mathrm{d}}{\mathrm{d} t} i+\left(\begin{array}{l}
X \\
Y
\end{array}\right)=M \frac{\mathrm{d}}{\mathrm{d} t} i+N
$$

Die Reaktion der elektrischen Maschine auf die angelegte Spannung setzt sich aus der Summe der Einzelreaktionen diskreten Scheiben zusammen. Der Strom ist für alle Scheiben gleich, erscheint jedoch im rotorfesten dq-Koordinatensystem bei Verdrehung der Scheibe $j$ durch Schrägung oder Torsion um den elektrischen Winkel $\Theta_{j}$ verdreht, so dass eine Rotation in ein gemeinsames Koordinatensystem durchgeführt wird.

$$
\begin{aligned}
& u=\sum_{j}^{n} u_{j}=\sum_{j}^{n} \operatorname{Rot}\left(\Theta_{j}\right)\left(M_{j} \frac{\mathrm{d}}{\mathrm{d} t} i+N_{j}\right) \\
& \text { mit } \operatorname{Rot}\left(\Theta_{j}\right)=\left[\begin{array}{rr}
\cos \left(\Theta_{j}\right) & -\sin \left(\Theta_{j}\right) \\
\sin \left(\Theta_{j}\right) & \cos \left(\Theta_{j}\right)
\end{array}\right]
\end{aligned}
$$

Zur Implementierung in der Simulation wird diese Gleichung nach der gesuchten Ableitung des Stromes umgestellt.

$$
\begin{aligned}
\frac{\mathrm{d}}{\mathrm{d} t} i= & {\left[\sum_{j}^{n}\left(\operatorname{Rot}\left(\Theta_{j}\right) M_{j} \operatorname{Rot}\left(\Theta_{j}\right)^{-1}\right)\right]^{-1} \ldots } \\
& \cdot\left[u-\sum_{j}^{n}\left(\operatorname{Rot}\left(\Theta_{j}\right) N_{j}\right)\right]
\end{aligned}
$$

\subsection{Kopplung der Domänen}

Die Feldberechnung liefert die elektromagnetischen Anregungskräfte an den FE-Knoten, welche nach der Eggshell-Methode berechnet werden [29], [30]. Diese müssen der EMKS in jedem Zeitschritt aufgeprägt werden. Da die Zeitkonstante der Strukturdynamik bei Betrachtung von Schwingungen im hörbaren Frequenzbereich (< $20 \mathrm{kHz}$ ) deutlich unter der der Schaltungssimulation liegt, ist analog zur Feld-Schaltungs-Berechnung eine Entkopplung zielführend.

Die elektrotechnische Domäne ist in Matlab/Simulink umgesetzt, die EMKS in SIMPACK. Beide verwenden einen Gleichungssystemlöser mit variabler Zeitschrittweite und „Zero-Crossing Detection", um trotz der nichtlinearen Schaltvorgänge bzw. Kontaktveränderungen der Struktur eine hohe Ausführungsgeschwindigkeit zu erreichen. Für ein paralleles Entwickeln der Teilbereiche bietet sich als Übergabeparameter der Strom an, das Zahnkraftkennfeld wird in der EMKS hinterlegt. Auf diese Weise ist die EMKS mit einem idealen Regler (durch Vorgabe einer festen dq-Stromkombination) ohne Stromberechnung ebenso lauffähig wie die Stromsimulation mit einer vorgegebenen Drehzahl oder einfachem Drehschwingermodell. Für eine effiziente Zusammenarbeit von Simulink und SIMPACK ist die Aktualisierung der Schnittstellen (Eingabe des Stroms, Ausgabe der Bewegung) mit einer festen Abtastrate erforderlich. Um Abtastartefakte (engl. Aliasing), welche aus Unterabtastung der hochfrequenten Anteile des Stroms durch die EMKS entstehen, zu reduzieren, muss der übergebene Strom mit geringem Phasenverzug tiefpassgefiltert werden. Geeignet ist z. B. ein Butterworth-Filter zweiter Ordnung (Eckfrequenz $20 \mathrm{kHz}$ ). Die Abtastrate der Schnittstellen muss als Kompromiss zwischen ausreichender Dämpfung des Filters und Ausführungsgeschwindigkeit gewählt werden (hier 60 kHz).

\subsection{Zahnkräfte}

Die Knotenkräfte aus der FE-Berechnung müssen für die modale Darstellung der EMKS mit einer begrenzten Anzahl von Kraftangriffspunkten aufbereitet und Aliasing bei der Kennfeldabtastung vermieden werden. Die Ordnungsreduktion aus [11] fasst die Knotenkräfte an jedem Zahn auf einen zentralen Kraftangriffspunkt zusammen und transformiert sie in ein zahnspezifisches Koordinatensystem mit tangentialer und radialer Komponente (vgl. Abb. 3) 


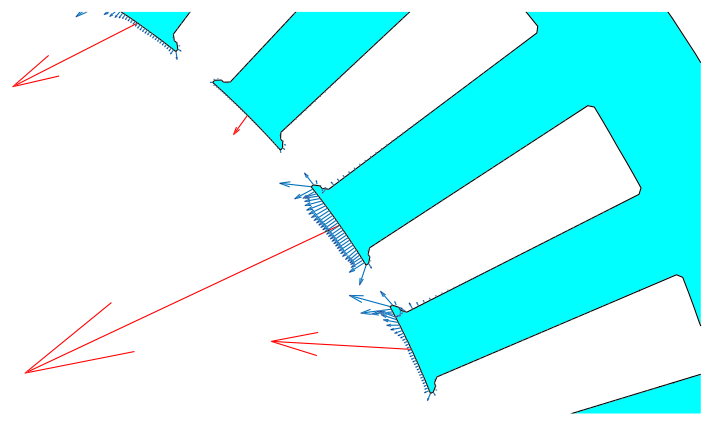

Abb. 3. Knotenkräfte (kl. Pfeile) und resultierende Zahnkraft (gr. Pfeile) am Stator, Rotor ausgeblendet

sowie Kippmoment (durch die Kraftverschiebung). Die unbekannte Axialkraft wird für die kontinuierlich geschrägte Maschine abgeschätzt aus dem Schrägungswinkel und der Annahme, dass die Tangentialkraft normal auf der Zahnflanke steht.

Aus den Schrittweiten des 5-dimensionalen Kraftkennfelds (Dimensionen Drehwinkel $\gamma$, Exzentrizität mit Radius $r_{E x}$ und Winkel $\phi$, Strom $i_{d}$ und $i_{q}$ ) ergeben sich die berücksichtigten Ordnungen, welche sich im Zusammenhang mit der Änderungsgeschwindigkeit des Parameters auf eine zeitliche Kraftfrequenz umrechnen lassen. Die Änderungsgeschwindigkeit des Stromes ist durch den Tiefpassfilter begrenzt und die Änderung der Exzentrizität aufgrund der Massenträgheit gering gegenüber der Änderung der Drehwinkelkoordinate, so dass letztere die dominante Dimension für hochfrequente Kraftanregungen darstellt. Durch Fourierzerlegung der DrehwinkelDimension können die hochfrequenten Ordnungen entfernt werden. Eine Rücktransformation in diskrete Kräfte in jedem Zeitschritt der EMKS schließt sich aufgrund des Rechenaufwands aus, so dass vorab ein diskretes, in Drehwinkeldimension höher aufgelöstes ( $>=$ Faktor 5) Kennfeld erstellt wird welches lediglich eine lineare Interpolation zwischen den Stützstellen erfordert.

Beim Nachschlagen der Zahnkräfte wird der Ist-Zustand der Simulation durch Rotation und Ausnutzung der Symmetrie auf einen äquivalenten, im Kennfeld vorhandenen Zustand zurück geführt. Die Nachschlagewerte (Index K) des Stromes $i_{d, K}$ und $i_{q, K}$ ergeben sich aus Rotation des globalen Stromes im resolverbezogenen $\mathrm{dq}$ Koordinatensystem in das Koordinatensystem der jeweiligen Scheibe. Da aufgrund der Statorsymmetrie das Kennfeld die Winkelkoordinate der Exzentrizität $\phi$ nur bis zum Wert der Nutteilung $\tau_{N}$ abbildet, wird sie bei Werten außerhalb dieses Bereichs mittels ModuloFunktion auf diesen zurück geführt, um $\phi_{K}$ zu erhalten.

$$
\phi_{K}=\phi \bmod \tau_{N}
$$

Folglich müssen die nachgeschlagenen Zahnkräfte den Zähnen neu zugeordnet werden, wenn der Exzentrizitätswinkel $\phi$ außerhalb des Kennfeldbereichs liegt. Dies entspricht einer Rotation der Zahnzuordnung um den ganzzahligen Wert der Zahnverschiebung $z$.

$$
z=\left\lfloor\frac{\phi}{\tau_{N}}\right\rfloor
$$

Analog muss der Drehwinkel des Rotors $\gamma$ auf den im Kennfeld vorhandenen Bereich bis $\gamma_{\max }$ zurück geführt werden. Weiterhin muss die Drehwinkelkoordinate zum Nachschlagen, $\gamma_{K}$, um die Verdrehung, welche die Zahnverschiebung verursacht, korrigiert werden. Daraus resultiert die Bedingung, dass die Drehwinkelschrittweite der FE-Berechnungen zu einem ganzzahligen Vielfachen der Nutteilung gewählt werden muss.

$$
\gamma_{K}=\left(\gamma-z \cdot \tau_{N}\right) \bmod \gamma_{\max }
$$

Bei Vernachlässigung von Magnetisierungsabweichungen genügt durch die Symmetrie des Rotors die Kennfeldberechnung von $\gamma$ über eine Polteilung. In diesem Fall ist zu berücksichtigen, dass die Kennfeldstützstelle mit $r_{E x}=0$ redundante Informationen enthält: Die Zahnkräfte wiederholen sich nach einer Polteilung, statt des Vollmodells wäre ein Teilmodell ausreichend. Dies kann bei numerischen Abweichungen (z. B. durch das FE-Netz) zwischen theoretisch gleichen Kräften zu einer Unstetigkeit des Kraftverlaufs bei $r_{E x}=0$ führen, welche zur Erhaltung der Stabilität der Simulation entfernt werden müssen.

Dem Kennfeld wird in den sich wiederholenden Dimensionen Rotordrehwinkel und Exzentrizitätswinkel am Rand die erste Stützstelle, welche entsprechend der Nachschlagevorschrift angepasst wird, als letzte Stützstelle angehangen, um den Nachschlageprozess ohne weitere Berücksichtigung des Kennfeldrandes umsetzen zu können.

\subsection{Elastische Mehrkörper-Simulation}

Im Rahmen der strukturdynamischen Modellbildung werden die Bauteile und Baugruppen in Form von (elastischen) Körpern und Koppelelementen (Kraftelemente und Gelenke) abstrahiert. Die elastischen Modellelemente werden unter Verwendung der FiniteElemente-Methode modelliert. Sie beinhalten vollflexible Gehäuse, Wellen und die Fluid-Struktur-Wechselwirkung von Statorgehäuse und Maschinenkühlmittel. Die strukturdynamischen Eigenschaften von Rotor und Stator, die sich durch einen geschichteten Aufbau auszeichnen, werden mit Hilfe von Materialmodellen mit richtungsabhängigen Eigenschaften modelliert und unter Verwendung von Methoden der Multi-Skalen-Modellierung (Repräsentatives Volumenelement - RVE) und Messungen parametriert. Zudem werden die lastabhängigen, nichtlinearen Steifigkeiten der Rillen- und Kegelrollenlager sowie das Anregungsverhaltendes Getriebes in dieser Umgebung eingebunden. Kinematische Kopplungen, d. h. Gelenke, kommen zur Abbildung der Tripoden- und Gleichlaufgelenke der Gelenkwellen zum Einsatz [18], [19], [31], [32], [33].

\section{Validierung und Ergebnisse}

Der Vergleich zwischen dem gemessenen und simulierten Strangstrom (Abb. 5) im Hochlauf bis $45001 /$ min (entsprechend $450 \mathrm{~Hz}$ Strom-Grundfrequenz) zeigt eine gute Übereinstimmung sowohl der Stromharmonischen der Maschine (Ordnung $-5,+7,-11,+13$, ...) als auch der PWM-Fächer bei PWM-Frequenz und ihren Vielfachen. Die Amplitude der PWM-Harmonischen fällt in der Simulation etwas geringer aus als in der Messung. Ein Erklärungsansatz ist das frequenzabhängige Verhalten der Maschineninduktivität, welche bei hohen Stromfrequenzen abnimmt und durch die als stationär angenommene Feldberechnung nicht abgebildet wird.

In Abb. 6 oben ist die Oberflächenbeschleunigung radial auf der elektrischen Maschine dargestellt, welche mit dem Systemmodell berechnet wurde. Der Vergleich zur Messung (unten) offenbart die gute Übereinstimmung der Anregungsordnungen, welche sowohl von elektrischer Maschine als auch den beiden Verzahnungsstufen des Getriebes stammen. In der Simulation weist der Rotor des Beispielantriebsstrangs eine sehr geringe dyn. Exzentrizität auf, so dass das Getriebe gegenüber der akustisch unauffälligen PMSM in diesem Fall das NVH-Verhalten dominiert. Pegelüberhöhungen durch Eigenfrequenzen, insbesondere im niedrigeren Frequenzbereich bis $5 \mathrm{kHz}$, werden in der Simulation gut abgebildet. Abweichungen sind im Campbell-Diagramm im Hintergrundpegel erkennbar, welcher bei der Messung breitbandig und über den gesamten Drehzahlbereich höher liegt, was u.a. auf Anregungen aus Reibung und der Getriebeölverwirbelung oder auf die Prüfstandsumgebung zurück geführt werden kann. Weiterhin zeigt der PWM-Fächer in der 


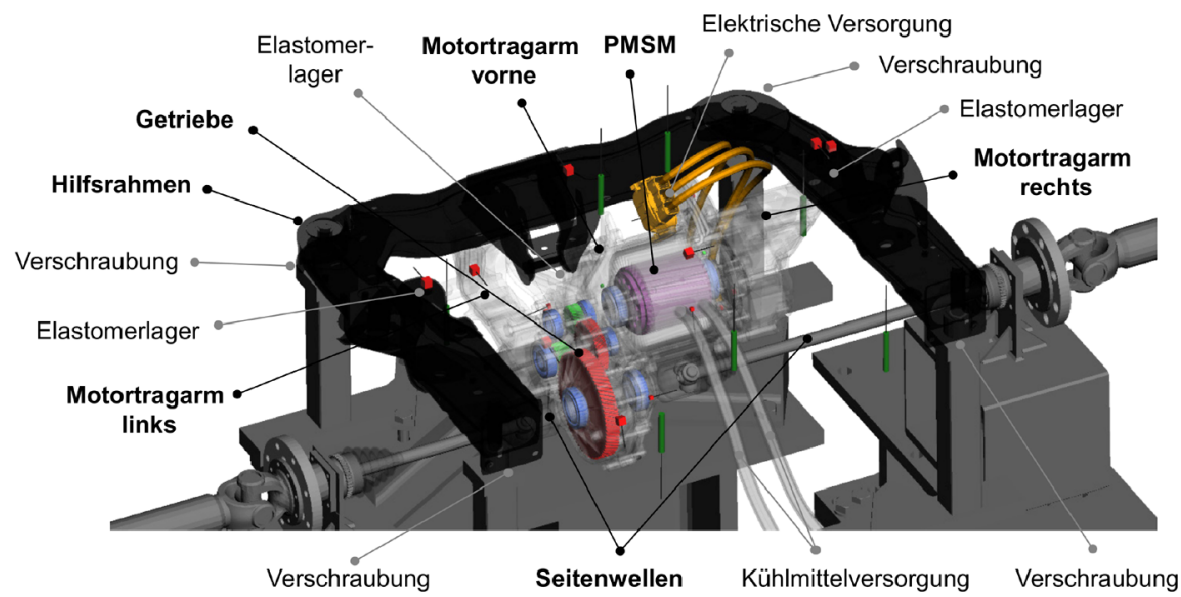

Abb. 4. Betrachteter Antriebsstrang zur Anwendung der Methode
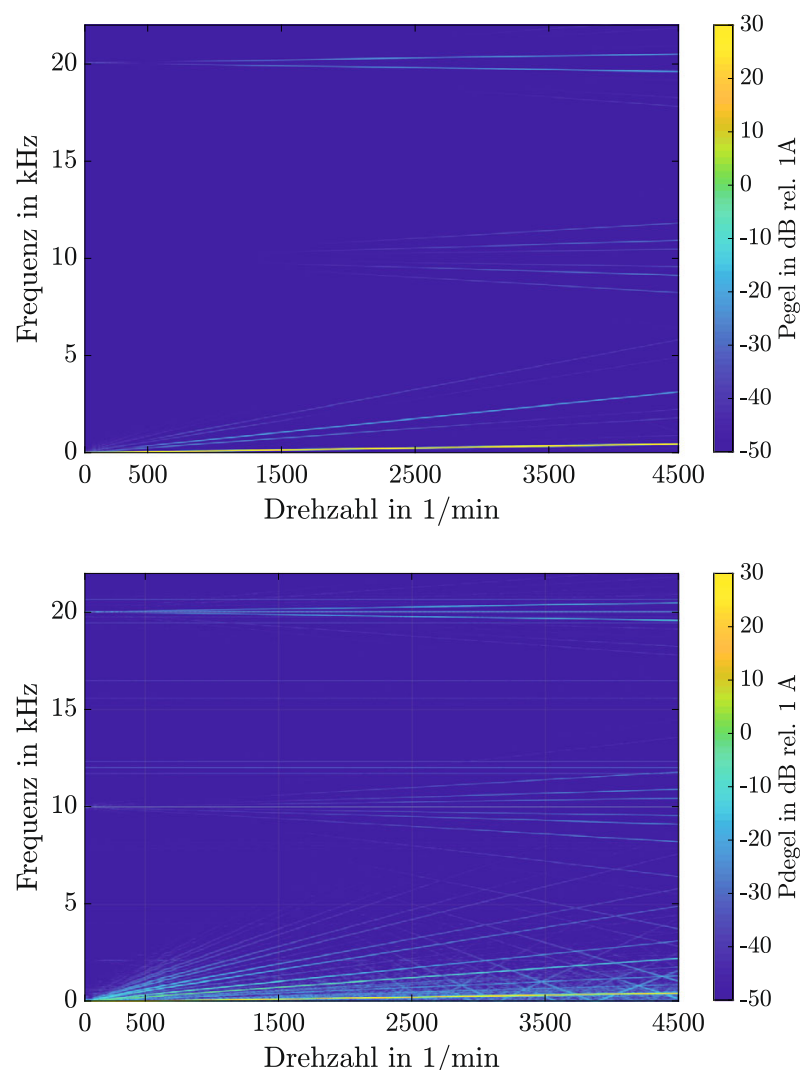

Abb. 5. Strangstrom, oben Simulation, unten Messung

Messung mehr Bänder, vermutet werden kann ein Zusammenhang zu den nicht modellierten parallelen Pfaden der Wicklung.

\subsection{Variantenrechnung}

Die hohe Ausführungsgeschwindigkeit ermöglicht die Simulation von Varianten, um die Sensitivität des NVH-Verhaltens bezüglich verschiedener Bauteile oder Effekte zu bewerten. Exemplarisch zeigt Abb. 7 die Simulation einer idealen el. Maschine ohne Exzentrizität und Magnetisierungsabweichungen. Die geringen Unterschiede im Vergleich zur Referenzsimulation (Abb. 6 oben) zeigen, dass das
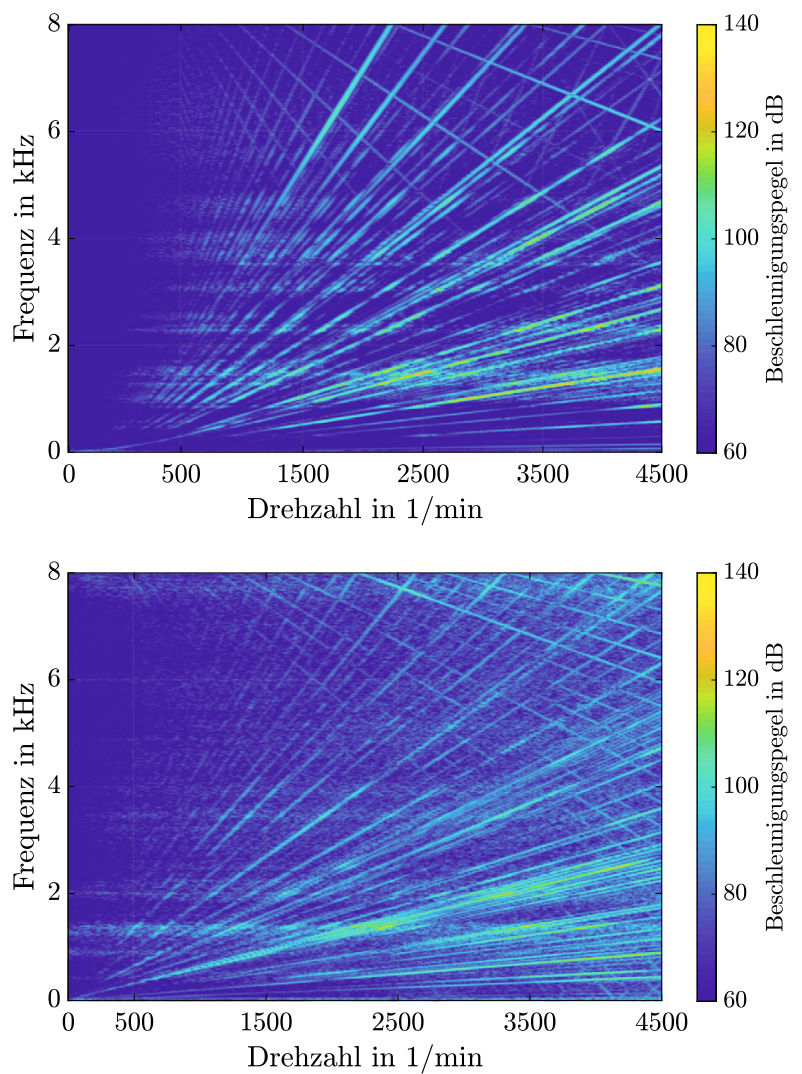

Abb. 6. Oberflächenbeschleunigung normal auf der el. Maschine. Oben Simulation, unten Messung

$\mathrm{NVH}$-Verhalten des betrachteten Antriebsstranges vom Getriebe dominiert wird, und daher für eine Verbesserung der Geräuschcharakteristik an diesem angesetzt werden müsste. Dies ist u.A. zurückzuführen auf die hohe Polzahl der el. Maschine, wodurch sich die Anregungskräfte mehr über den Umfang verteilen als z. B. bei einer Bruchlochwicklung.

In Abb. 8 ist eine weitere Variantenrechnung dargestellt, in welcher die modalen Dämpfungen aller Moden der Gehäusebaugruppe auf $2 \%$ eingestellt wurden. Für die meisten Moden bedeutet das ei- 


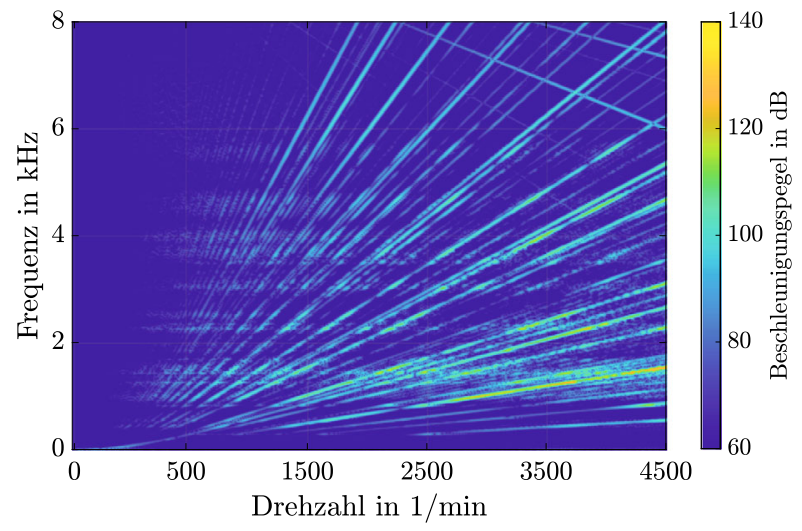

Abb. 7. Oberflächenbeschleunigung normal auf der el. Maschine. Simulation der el. Maschine ohne Exzentrizität und ohne Magnetisierungsabweichungen

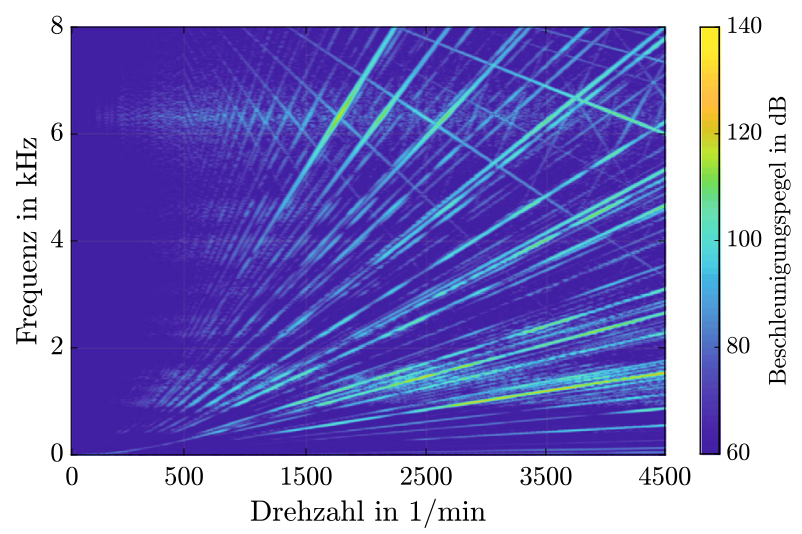

Abb. 8. Oberflächenbeschleunigung normal auf der el. Maschine. Simulation für eine modale Dämpfung von $2 \%$ für alle Moden der Gehäusebaugruppe

ne Erhöhung (gemessener Mittelwert 0,4 \%). Die Gehäusebaugruppe umfasst das Gehäuse der el. Maschine und des Getriebes sowie die Motortragarme, d.h. die in Abb. 4 halbtransparent dargestellten Komponenten. Erwartungsgemäßliegen die Körperschallpegel im Durchschnitt etwas niedriger, im Gegensatz dazu ist bei $6,4 \mathrm{kHz}$ eine in der Realität stark gedämpfte Mode nun erkennbar. Die Unterschiede zur Referenzsimulation sind im Allgemeinen gering, und deuten darauf hin dass für die Berechnung der NVH-Charakteristik Abweichungen der Dämpfungswerte von untergeordneter Bedeutung sind.

\section{Zusammenfassung und Ausblick}

In der Entwicklung stellt die vorrangige Optimierung des $\mathrm{NVH}$ Verhaltens aufgrund von Zielkonflikten mit Kosten oder Wirkungsgrad den Ausnahmefall dar. Relevanter ist die gezielte Lösung abgegrenzter Schwingungsprobleme möglichst kosteneffizient durch Ausregelung oder Einzelteiloptimierung. Das vorgestellte Verfahren erlaubt beides, und ermöglicht darüber hinaus ein tieferes Systemverständnis des NVH-Verhaltens, in dem beispielsweise die Zusammenhänge zwischen Drehmomentripple der el. Maschine und den Verzahnungsanregungen am validierten Systemmodell untersucht werden können. Die Methodik basiert auf etablierten Berechnungs- ansätzen und ist für eine hohe Ausführungsgeschwindigkeit möglichst parallelisierbar auf moderne Hardware ausgelegt.

\section{Danksagung}

Open Access funding provided by Projekt DEAL. Das Forschungsvorhaben wurde im Rahmen des Programms zur Förderung der industriellen Gemeinschaftsforschung (IGF-Nr. 18764 N) vom Bundesministerium für Wirtschaft und Energie (BMWi) über die Arbeitsgemeinschaft industrieller Forschungsvereinigungen (AiF) e.V.

Gefördert durch

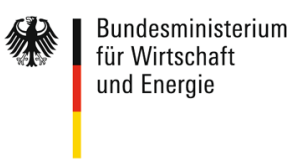

aufgrund eines Beschlusses des Deutschen Bundestages Deutschen Bundestages gefördert. Die Autoren danken weiterhin der Forschungsvereinigung Antriebstechnik e.V. (FVA) für die Durchführung und fachliche Unterstützung durch Experten aus dem FVA Netzwerk, sowie der Deutschen Forschungsgemeinschaft (DFG) für die Förderung der interdisziplinären Forschung im Rahmen des Graduiertenkollegs mobilEM (GRK 1856).

Hinweis des Verlags Der Verlag bleibt in Hinblick auf geografische Zuordnungen und Gebietsbezeichnungen in veröffentlichten Karten und Institutsadressen neutral.

Open Access Dieser Artikel wird unter der Creative Commons Namensnennung 4.0 International Lizenz veröffentlicht, welche die Nutzung, Vervielfältigung, Bearbeitung, Verbreitung und Wiedergabe in jeglichem Medium und Format erlaubt, sofern Sie den/die ursprünglichen Autor(en) und die Quelle ordnungsgemäß nennen, einen Link zur Creative Commons Lizenz beifügen und angeben, ob Änderungen vorgenommen wurden. Die in diesem Artikel enthaltenen Bilder und sonstiges Drittmaterial unterliegen ebenfalls der genannten Creative Commons Lizenz, sofern sich aus der Abbildungslegende nichts anderes ergibt. Sofern das betreffende Material nicht unter der genannten Creative Commons Lizenz steht und die betreffende Handlung nicht nach gesetzlichen Vorschriften erlaubt ist, ist für die oben aufgeführten Weiterverwendungen des Materials die Einwilligung des jeweiligen Rechteinhabers einzuholen. Weitere Details zur Lizenz entnehmen Sie bitte der Lizenzinformation auf http://creativecommons.org/licenses/by/4.0/deed.de

\section{Literatur}

1. Fritze, H. (1921): Über die Geräuschbildung bei elektrischen Maschinen. Arch. Elektrotech., 10(3-4), 73-95

2. Sequenz, H. (1932): Die Wahl der Nutenzahlen bei Käfigankermotoren. Elektrotechn. Masch.-Bau, 50, 428-434.

3. Jordan, H. (1950): In Geräuscharme Elektromotoren, Essen, Giradet

4. Pyrhoönen, J., Jokinen, T., Hrabovcova, V. (2008): Design of rotating electrical machines. United Kingdom, Chichester: John Wiley \& Sons Ltd.

5. Habetler, T. G., Divan, D. M. (1991): Acoustic noise reduction in sinusoidal PWM drives using a randomly modulated carrier. IEEE Trans. Power Electron., 6, 356-363.

6. Haaf, D., Johnen, M., Gold, P. W., Henneberger, G. (2003): Einfluss des Umrichterbetriebs auf das akustische Verhalten im drehzahlvariablen Gesamtsytem Drehstrommotor-Getriebe. FVA Forschungsheft 699, Forschungsvorhaben 309 II. Forschungsvereinigung Antriebstechnik e.V. (FVA), erhältlich auf Anfrage.

7. Gieras, J. F., Wang, C., Cho Lai, J. (2006): Noise of polyphase electric motors. New York: CRC Press (Taylor \& Francis Group).

8. Zeller, P. (2012): Handbuch Fahrzeugakustik. Grundlagen, Auslegung, Berechnung, Versuch. 2. überarbeitete Aufl. Wiesbaden: Vieweg+Teubner Verlag.

9. Genuit, K. (2010): In Sound-Engineering im Automobilbereich. Methoden zur Messung und Auswertung von Geräuschen und Schwingungen, Berlin, Heidelberg: SpringerVerlag Berlin Heidelberg.

10. Valavi, M., Nysveen, A., Nilsen, R., Le Besnerais, J., Devillers, E. (2017): Analysis of magnetic forces and vibration in a converter-fed synchronous hydrogenerator. In IEEE energy conversion congress and exposition (ECCE).

11. Herold, T., Franck, D., Böhmer, S., Schröder, M., Hameyer, K. (2015): Transientes Simulationsmodell für lokale Kraftanregungen elektrischer Antriebe. E\&l, Elektrotech. Inf.tech., 132(1), 46-54.

12. Knaus, O., Schneider, J., Klarin, B. (2018): Simulation in der Entwicklung automotiver E-Maschinen. ATZ Extra (S. 32-37). Berlin: Springer. 05/2018. 
13. Franck, D., Herold, T., Hameyer, K. (2013): Transient acoustic simulations of electrical drive-trains. In Conference on acoustics. AIA-DAGA 2013.

14. Bambton, M. C. C., Craig, R. R. Jr. (1968): Coupling of substructures for dynamic analyses. AIAA J., 6(7), 1313-1319.

15. Ewins, D. J. (2000): Modal testing: theory, practice and application. 2. ed. England: Research Studies Press Ltd.

16. Avitabile, P. (1998-2014): Back to basics: modal space. Our own little world. Article series. Springer.

17. Schwarzer, M. (2017): Structural dynamic modeling and simulation of acoustic sound emissions of electric traction motors. Ph.D. Dissertation, TU Darmstadt.

18. Rick, S., Wegerhoff, M., Klein, J., Hameyer, K., Jacobs, G., Vorländer, M. (2015): EMotive NVH-Simulationsmodell: Modellbildung zur NVH-Simulation eines E-Motive Antriebsstrangs. FVA Forschungsheft 1130, Forschungsvorhaben 682 I. Forschungsvereinigung Antriebstechnik e.V. (FVA), erhältlich auf Anfrage.

19. Wegerhoff, M. (2017): Methodik zur numerischen NVH Analyse eines elektrifizierten PKW Antriebsstrangs. Ph.D. Dissertation, RWTH Aachen University.

20. Herold, T., Lange, E., Hameyer, K. (2011): System simulation of a PMSM servo drive using field-circuit coupling. IEEE Trans. Magn., 47(5), 938-941.

21. Herold, T., Franck, D., Lange, E., Hameyer, K. (2011): Extension of a D-Q model of a permanent magnet excited synchronous machine by including saturation, cross-coupling and slotting effects. In Electric machines \& drives conference (IEMDC). New York: IEEE International.

22. Frohne, H. (1968): Über den einseitigen magnetischen Zug in Drehfeldmaschinen. Arch. Elektrotech., 51(5), 300-308.

23. Jordan, H., Schroeder, R.-D., Seinsch, H. O. (1981): Zur Berechnung einseitig magnetischer Zugkräfte in Drehfeldmaschinen. Arch. Elektrotech., 63(2) 117-124.

24. Zhang, M., Macdonald, A., Tseng, K.-J. Burt, G. M. (2013): Magnetic equivalent circuit modeling for interior permanent magnet synchronous machine under eccentricity fault. In Power engineering conference (UPEC) (S. 1-10)
25. Lundin, U., Wolfbrandt, A. (2009): Method for modeling time-dependent nonuniform rotor/stator configurations in electrical machines. IEEE Trans. Magn., 45(7), 2009.

26. Piriou, F., Razek, A. (1990): A model for coupled magnetic-electric circuits in electric machines with skewed slots. IEEE Trans. Magn., 26(2), 1096-1100.

27. Williamson, S., Flack, T. J., Volschenk, A. F. (1994): Representation of skew in timestepped two-dimensional finite-element models of electrical machines. IEEE Ind. Appl., 31(5), 1009-1015

28. Jaeger, M., Rick, S., Hameyer, K. (2018): Current simulation of a controlled PMSM including skew and torsional rotor vibrations. In 2018 XIII international conference on electrical machines (ICEM), Griechenland.

29. Henrotte, F., Felden, M., van der Giet, M., Hameyer, K. (2019): Electromagnetic force computation with the Eggshell method. Proceedings of the 14th international symposium on numerical field calculation in electrical engineering. Graz: IGTE.

30. van der Giet, M., Franck, D., Henrotte, F., Hameyer, K. (2010): Comparative study of force computation methods for acoustic analyses of electrical machines. In Proceedings of the 14th international symposium on numerical field calculation in electrical engineering, IGTE.

31. Drichel, P., Jaeger, M., Müller-Giebeler, M. (2019): Mit elektrischem Antrieb und modellbasierter Systemanalyse nahezu lautlos in die Zukunft. ATZ Extra, 2019/24 (Suppl 5). Wiesbaden: Springer.

32. Drichel, P., Wischmann, S., Berroth, J., Jacobs, G. (2019): Modellierungsmethodik zur multiaxialen Abbildung des höherfrequenten Übertragungsverhaltens von Elastomerlagern in der NVH-Systemsimulation. Antriebstechnisches Kolloquium (ATK) 2019, Tagungsband 18

33. Drichel, P., Berroth, J., Jacobs, G. (2018): Multi-domain simulation for the assessment of the NVH-behavior of electric vehicles with special attention to the influence of rotor eccentricity. In Dassault user conference 2018

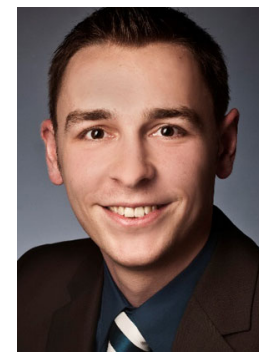

\section{Michael Schröder}

studierte an der RWTH Aachen Elektrotechnik und schloss im Mai 2012 mit dem Diplom ab. Im Anschluss wurde er wissenschaftlicher Mitarbeiter am Institut für Elektrische Maschinen der RWTH Aachen. Von Okt. 2014 bis Okt. 2017 war er Gruppenleiter der Gruppe Analyse und Design mit Forschungsthemen im Bereich der weich- und hartmagnetischen Materialien, Isolierstoffsystemen sowie dem strukturdynamischen und akustischen Verhalten el. Maschinen. Seit Nov. 2017 ist er ein Oberingenieur des Instituts. Seine Forschungsschwerpunkte liegen im Bereich des akustischen und strukturdynamischen Verhaltens elektrischer Maschinen unter dem Einfluss von Fertigungsabweichung.

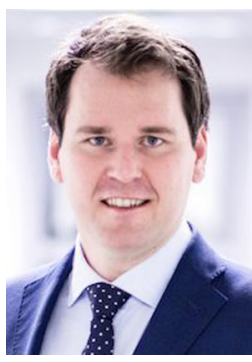

\section{Joerg Berroth}

studierte Maschinenbau an der RWTH Aachen. Nach seinem Studium war er von 2012 bis 2014 als wissenschaftlicher Mitarbeiter im Bereich Antriebstechnik in der Forschungsgruppe Simulation am iMSE tätig. Zu seinen Forschungsschwerpunkten gehörte die Analyse der Antriebsstrangdynamik von Maschinen, im speziellen Windenergieanlagen. Von 2014 an führte er seine Arbeiten am Center for Wind Power Drives (CWD) fort. Dort leitete er bis Aug. 2017 die Forschungsgruppe Modellierung und Simulation im Bereich Systemdesign mit dem Schwerpunkt in der Weiterentwicklung von Simulationsmethoden auf Basis der EMKS. Nach erfolgreicher Promotion 2017 kehrte er ans MSE zurück und leitet dort seitdem den Bereich Systems Engineering - Modelling and Simulation. 


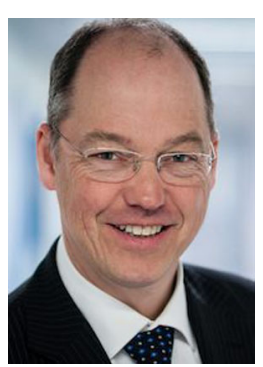

\section{Georg Jacobs}

studierte Maschinenwesen mit Vertiefungsrichtung Konstruktionstechnik an der RWTH Aachen und promovierte 1993 am IHP zum Verschleißverhalten hydraulischer Maschinen. Nach dreijähriger Mitarbeit in der Institutsleitung wechselte er 1996 in die Industrie zur Firma Müller Weingarten. 1997 trat er in die Mannesmann Rexroth AG, heute Bosch Rexroth $A G$, ein, wo er unterschiedliche technische Leitungsfunktionen innehatte, bevor er 2006 als Mitglied der Geschäftsbereichsleitung Hydraulik Verantwortung für die Antriebstechnik bei Bosch Rexroth übernahm. Seit Okt. 2008 ist Prof. Jacobs Leiter des Lehrstuhls und Instituts für Maschinenelemente und Maschinengestaltung (IME). Seit 2013 ist er an der RWTH Institutsleiter des neu gegründeten Chair for Wind Power Drives sowie Sprecher des Vorstandes des ebenfalls neu gegründeten Center for Wind Power Drives. Von 2012 bis 2014 war Prof. Jacobs Prodekan für Struktur und von 2014 bis 2016 Dekan der Fakultät für Maschinenwesen. Seit 2016 ist er Professor und Institutsleiter am Institut für Allgemeine Konstruktionstechnik des Maschinenbaus (IKT), welches 2018 mit dem IME zum von ihm geleiteten Institut für Maschinenelemente und Systementwicklung (MSE) zusammengeführt wurde.

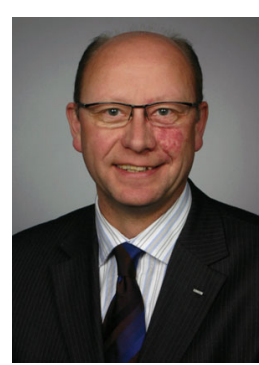

Kay Hameyer

erwarb sein Diplom in Elektrotechnik von der Universität Hannover und promovierte an der Technischen Universität Berlin. Nach seinem Studium arbeitete er für die Robert Bosch GmbH in Stuttgart als Entwicklungsingenieur für permanentmagneterregte Servomotoren und Kraftfahrzeugbordnetzkomponenten. Bis 2004 war er Professor für numerische Feldberechnungen und elektrische Maschinen an der KU Leuven in Belgien. Seit 2004 ist er Professor und Leiter des Instituts für Elektrische Maschinen der RWTH Aachen. 2006 war er Vizedekan der Fakultät für Elektrotechnik und Informationstechnik von 2007 bis 2009 Dekan der Fakultät. Seine Forschungsschwerpunkte liegen im Bereich der numerischen Feldberechnung und -optimierung, dem Entwurf und der Regelung elektrischer, insbesondere permanentmagneterregter Maschinen und Drehfeldmaschinen. Seit vielen Jahren beschäftigt sich die Arbeit von Prof. Hameyer mit der elektromagnetischen Geräuschanregung elektrischer Antriebe und der Charakterisierung ferromagnetischer Materialien und Isolierstoffen. Er ist Autor von mehr als 350 Journal-Publikationen, mehr als 700 internationalen Konferenzveröffentlichungen und vier Büchern, sowie Mitglied im VDE, IEEE Senior Member und Fellow des IET. 